\title{
GPR system onboard a UAV for non-invasive detection of buried objects
}

\author{
Maria Garcia-Fernandez*, Yuri Alvarez-Lopez*, Borja Gonzalez-Valdes ${ }^{\dagger}$, Yolanda Rodriguez-Vaqueiro ${ }^{\dagger}$, \\ Ana Arboleya-Arboleya ${ }^{\ddagger}$, Fernando Las Heras* and Antonio Pino ${ }^{\dagger}$ \\ *Area of Signal Theory and Communications, University of Oviedo, Gijón (Spain). Email: mariagarcia@tsc.uniovi.es \\ ${ }^{\dagger}$ Dept. of Signal Theory and Communications, University of Vigo, Vigo (Spain). \\ ${ }^{\ddagger}$ Area of Signal Theory and Communications, University Rey Juan Carlos, Madrid (Spain).
}

\begin{abstract}
In this contribution, a novel subsurface imaging system to detect buried targets is presented. It is mainly composed by a Ground Penetrating Radar (GPR) mounted onboard an Unmanned Aerial Vehicle (UAV). Thus, the system can be used to inspect areas without being in contact with the soil, which makes it useful for detecting dangerous objects, such as landmines. The system architecture and some of the first prototype measurements, which are post-processed using a Synthetic Aperture Radar (SAR) algorithm, are presented in this contribution.
\end{abstract}

\section{INTRODUCTION}

Non-Invasive Testing (NDT) techniques are of great interest for the detection and imaging of buried targets in a wide range of applications such as security and defense (e.g. landmine detection), civil engineering (e.g. road inspection), archeology, and search and rescue operations. Ground Penetrating Radar (GPR) [1] has been considered one of the best techniques for subsurface imaging due to its capability to detect both metallic and dielectric targets, as well as providing electromagnetic images of the underground.

There are mainly two kinds of GPR systems: Forward Looking GPR (FLGPR), where the antennas are placed looking ahead of the vehicle at a standoff distance [2], and Down Looking GPR (DLGPR), where the antennas are perpendicular to the soil interface [3]. The former presents lower resolution, whereas the latter suffers from stronger reflections from the ground surface (clutter).

For some applications, such as landmine detection, the system must keep a safety distance from the inspected area. One approach to solve this challenge is to use lightweight terrestrial robots, with a low detonation risk but at a slow scanning speed. In order to minimize the risk and increase the speed, the approach presented in this contribution is based on a GPR mounted onboard an Unmanned Aerial Vehicle (UAV). In the literature, there are some systems for detecting buried targets using UAVs [4],[5]. Nevertheless, these systems cannot generate a high resolution radar image using Synthetic Aperture Radar (SAR) algorithms, since their positioning system is not accurate enough. Thus, small objects close to each other cannot be distinguished and the shape of the objects is not properly recovered. The system proposed in this contribution addresses this problem (i.e. the measurements can be coherently combined to obtain underground images).

\section{SyStem ARCHITECTURE}

The proposed system consists of a GPR onboard a UAV (shown in Fig. 1). The idea is that the UAV autonomously follows a predefined path over the inspected area, sending the geo-referred radar measurements to a ground control station where they are postprocessed to generate radar images of the subsurface. Thus, the system does not need to be in contact with the soil and it makes possible the detection of both metallic and dielectric targets (thanks to the use of a radar instead of a metal detector). It must be noticed that, in order to obtain high resolution images, radar measurements must be coherently combined. Therefore, positioning accuracy should be higher than a quarter wavelength.

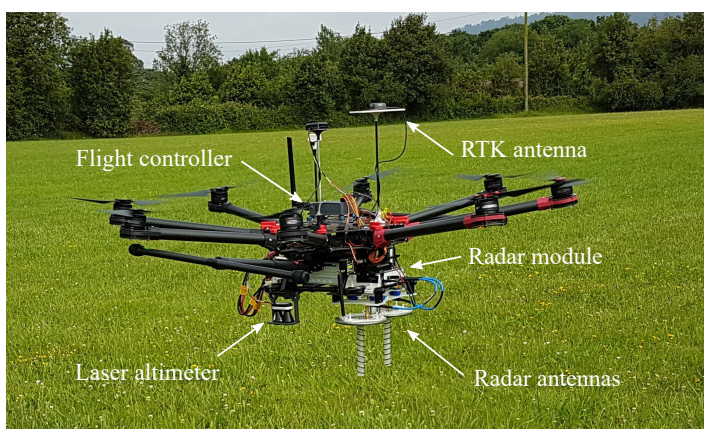

Fig. 1. GPR system onboard the UAV.

The prototype is composed by the following subsystems:

- Flight control subsystem, which consists of a flight controller and common positioning sensors (Inertial Measurement Unit -IMU-, barometer and Global Navigation Satellite System -GNSS- receiver).

- Communication subsystem.

- Accurate positioning subsystem to provide cm-level accuracy. It includes a Real Time Kinematic (RTK) system and a laser altimeter. The RTK system makes use of two RTK beacons: one on the UAV and another on the ground at a fixed position. The latter sends GNSS corrections to the former in order to achieve cm-level accuracy.

- Radar subsystem. An impulse radar working at $\mathrm{C}$ band (from 3.1 to $5.1 \mathrm{GHz}$ ) is used, achieving a $7.5 \mathrm{~cm}$ range resolution at the expense of a low penetration depth.

- Ground control station, where the geo-referred radar measurements are post-processed. 
It must be remarked that the radar subsystem is connected to the flight controller, so that geo-referred measurements are sent in real time to the ground control station.

Regarding the methodology, radar measurements are combined using a SAR algorithm. Assuming a quasi-monostatic configuration where the scattered field $E_{\text {scatt }}$ is measured on $\mathrm{M}$ acquisition points at $\mathrm{N}$ frequencies, the reflectivity at a point $\boldsymbol{r}^{\prime}$ is given by $(1)$, where $\boldsymbol{r}_{m}$ is the position of the m-th measurement and $k_{0, n}$ is the free-space wavenumber at frequency $f_{n}$. In order to mitigate the clutter, the average of all measurements is subtracted from each measurement (in the time domain) prior to the SAR processing.

$$
\rho\left(r^{\prime}\right)=\sum_{n=1}^{N} \sum_{m=1}^{M} E_{\text {scatt }}\left(f_{n}, \boldsymbol{r}_{m}\right) e^{j 2 k_{0, n}\left\|\boldsymbol{r}_{\boldsymbol{m}}-\boldsymbol{r}^{\prime}\right\|_{2}}
$$

\section{EXPERIMENTAL VALIDATION}

Experimental validation has been carried out in several stages: first, the radar module was tested in a controlled environment (a planar measurement range) and in a sandy beach; then, measurements were taken also in a sandy beach using all the prototype components; and, finally, several flights have been done to test the whole system. Some imaging results of the last two experiments are presented in this contribution.

\section{A. Ground testing}

Measurements using the prototype components were taken with a manual positioning scanner that allows to perform cross-range scanning. A 9-cm radius metallic disc was buried $15 \mathrm{~cm}$ deep in a sandy beach. The SAR image obtained is shown in Fig. 2, where the soil interface and the target are clearly distinguishable. It must be noticed that the target is detected deeper (at $27 \mathrm{~cm}$ depth), since the permittivity of the soil is not taken into account to obtain the SAR image.

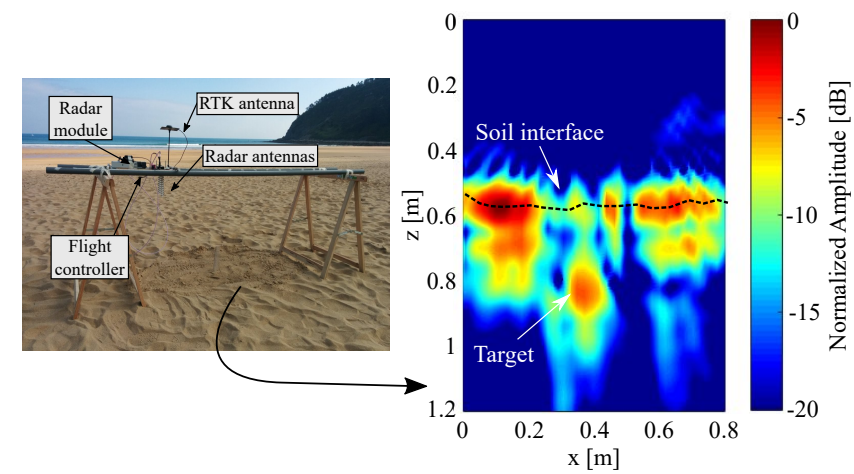

Fig. 2. Validation in a sandy beach: measurement setup on the left and SAR image of a buried metallic target on the right.

\section{B. Initial flight testing}

Once the prototype components were successfully tested, first in-flight tests were performed at the airfield shown in Fig. 1. First, a metallic bar (1 $\mathrm{m}$ long and $6 \mathrm{~cm}$ wide) was placed over the grass, perpendicular to the flight path. In the SAR image obtained (Fig. 3(a) , the bar is clearly detected.
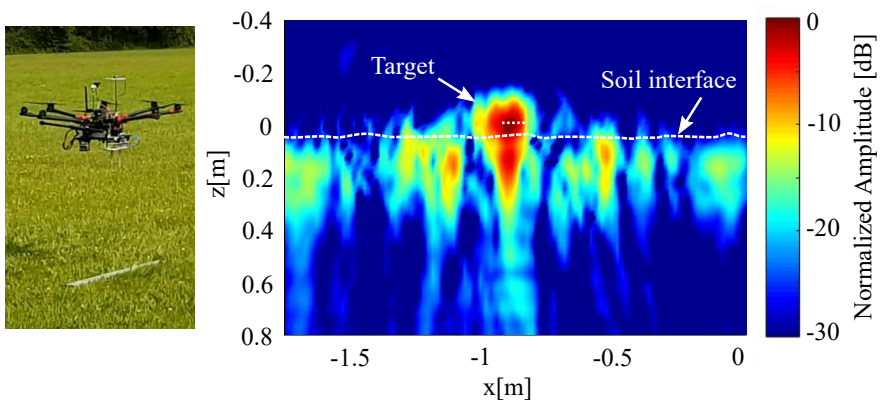

(a) Target over the ground
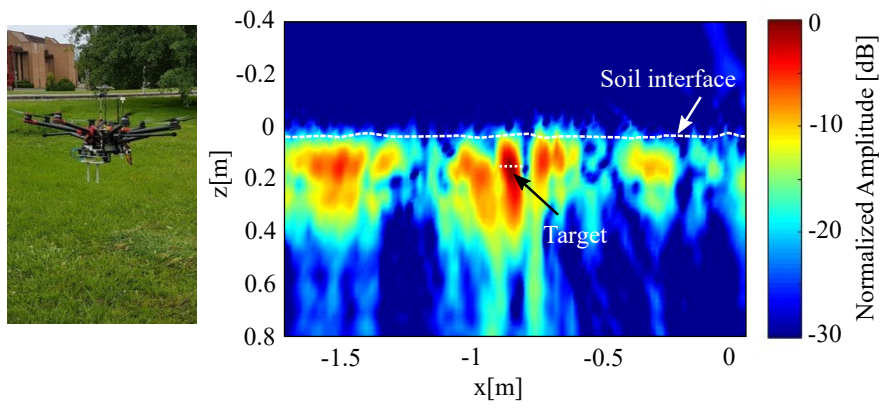

(b) Target buried

Fig. 3. Initial flight tests: setups on the left and SAR images on the right.

It can be noticed that the reflection coming from the soil is higher a few centimeters deeper than the grass level due to the higher water content at this depth. Then, for a second test, the bar was buried $5 \mathrm{~cm}$ below the soil interface. In this case, the bar is also detected in the SAR image (Fig. 3(b)), although the contrast with the soil reflections is smaller.

\section{CONCLUSION}

A novel system to detect buried targets based on integrating a GPR radar on a UAV has been presented in this contribution. It improves existing systems in several aspects such as speed, cost, security, and ability to detect both metallic and dielectric targets. The initial validation of the prototype is shown in this contribution for detecting metallic targets, and further validation for dielectric targets will be presented at the conference.

\section{ACKNOWLEDGEMENT}

This work has been supported by Government of Spain (under projects TEC2014-55290-JIN, TEC2015-73908JIN and grant FPU15/06341), Government of Asturias (IDI/2017/000095) and Galician Government (under project GRC2015/018) and under agreement for funding AtlantTIC.

\section{REFERENCES}

[1] D. J. Daniels, Ground Penetrating Radar, 2nd ed. London: IET, 2004.

[2] Y. Fuse, et al., "A null steering method for detecting buried objects with forward-looking GPR", in Proc. of EuCAP, Paris, March 2017.

[3] E. M. Rosen, et al., "Assessment of down-looking GPR sensors for landmine detection", in Proc. of the SPIE, vol. 5794, pp. 423-434, 2005.

[4] J. Colorado, et al., "An integrated aerial system for landmine detection: SDR-based ground penetrating radar onboard an autonomous drone", Advanced Robotics, vol. 31, pp. 791-808, 2017.

[5] L. G. Stolarczyk, et al., "Aerial electronic detection of surface and underground threats", US Patent 7336079, 2008. 